\title{
Studio degli accelerogrammi ottenuti nei recenti eventi sismici dell'Italia Centrale
}

\author{
(On the study of accelerograms obtained during the last seismic events \\ of Central (ialy)
}

\author{
R. Consola - F. Peronaci
}

Ricevuto il 23 Settembre 1971

\begin{abstract}
Riassunto, - Si espongono aleune consjderazioni riguarlanti l'analisi delle registrazioni ottenibili con gli accelerografi M.O. 2. Le medesime considerazioni vengono messe in pratiea su quattro terremoti effettivamente registrati ottenendone alcune grandezze fondamentali e gli spettri di velocità. Questi ultimi vengono poi mediati e tralotti in spettri di aceelerazione.

I risultati dimostrano la validita lell'uso del calcolatore elettronico per questo tipo di studi $\theta$ confermano l'importanza dell'installazione di una rete accelerometrica nazionale.
\end{abstract}

SUMmary. - Some considerations about the analysis of records obtainable by M.O. 2 accelerographs are exposed. Tlie same considerations are put into practice on four earthquakes really recorded, obtaining some fundamental constants and the velocity spectra. The latter are then averaged and translated in acceleration spectra.

The results slow the validity of using an electronic computer for these stulies and confirm the importance of a national accelerometric network.

1 - In seguito all'installazione di apparecchiature accelerometriche (M.O. 2) in prossimità dell'epicentro di zone colpite da terremoti, si sono ottenute registrazioni di repliche aventi intensità superiori al 
IV-V. In particolare sono state registrate tre leggere scosse a Mignano Iontelungo (di cui una da parte del (CN.E.N.) ed una piǹ forte a Valfabbrica (Perugria). Nessuna registrazione è stata ottenuta a 'Tuscania sebbene l'I.N.G. vi avesse installato uno strumento sin dal pomeriggio seguente la scossa principale.

\section{1. - DETERMINAZIONE DEJLE ACCEJERAZIONI.}

Come è noto, gli accelerografi M.O. 2 registrano su pellicola fotografira da $35 \mathrm{~mm}$ non perforata. Ta sensibilità per l'accelerazione è pari a circa $1,5 \mathrm{~cm} / \mathrm{g}$ per le due componenti orizzontali, mentre la soglia di scatto è di circa $0,01 \mathrm{~g}$; pertanto i terremoti di intensità appena suffciente ad avviare il meccanismo generano una traccia con ondulazioni di porhi clecimi di millimetro; questo si è verificato per le tre seosse registrate a Mignano.

('ome si ì già accennato in un precedente articolo, nel tentativo di utilizzare registrazioni di cosi scarsa entità si è proceduto ad ingrandire fotograficamente ( 5 volte) il tratto di pellicola contenente l'inizio tlella registrazione per una durata di circa 4 secondi, periodo nel quale sono comprese le onde di massima ampiezza. Dato che non si disponeva di una attrezzatura automatica, su queste riproduzioni fotografiche si sono lette le quote dei singoli punti della traccia, ad intervalli di un millimetro, cercanilo di apprezzare il decimo di millimetro. I'intervallo scelto sull'asse dei tempi corrisponde a circa $1 / 80$ di secondo, il che ci consente di rilevare componenti di frequenza inferiore a $40 \mathrm{~Hz}$. Si è constatato che un lettore sufficientemente esercitato può portare a termine l'analisi dei 300 punti che compongono l'accelerogramma in meno di mezz'ora. Successivamente i dati sono stati riportati su scherie perforate.

Ije quote dei punti sono state rilevate rispetto ad una linea di zero arbitraria, pertanto occorreva tradurle in accelerazioni vere, come se queste fossero misurate rispetto alla posizione di riposo diella traccia. Questo è un problema fondamentale nell'analisi degli accelerogrammi.

Si è subito scartato il metodo di ricavare direttamente dall'accelerogramma la linea di zero, dato ele questo porta ad errori dello stesso ordine di grandezza delle oscillazioni che si intendono misurare. Si is scelto percio il metodo analitico dei minimi quadrati. In pratica il metodo consiste nel determinare, rispetto al reticolo usato come riferimento, l'equazione ciella retta che rende minima la somma dei quadrati 
degli scarti tra i punti sperimentali e la retta cereata. L'intero calcolo viere rapidamente svolto dall'elaboratore elettronico, e quindi i valori che interessano vengono determinati per differenza dalla retta in questione e moltiplicati per l'opportuno fattore di scala.

Col metodo descritto si compensa automaticamente l'errore che iI lettore compie nel valutare il parallelismo tra il reticolo e la linea di ferie, errore che, come si is potuto constatare successivamente, idell'ordine di due o tre decimi di millimetro sull'intero accelerogramma.

\section{2. - TETERMINAZIONE DELIE GRANDEZZE FONDAMENTALI DEL.LA $\operatorname{scoss} A$.}

Il passo successivo delle nostre elaborazioni consiste nel determinare l'accelerazione massima e la potenza del terremoto. Quest'ultima, definita come

$$
P=\int_{0}^{\infty} a^{2}(t) d t,
$$

fove $a(t)$ ì l'accelerazione istantanea, viene in realtà calcolata come somma dei quadrati delle accelcrazioni ottennte punto per punto (vedi paragrafo 1).

I valori di queste grandezze sono stati riportati nella Tabella I e messi a confronto con la magnituro (determinata con gli strumenti campione dell'Osservatorio Sismico di Monteporzio Catone) e l'intensità macrosismica rilevata sul Iuogo.

Tabella I

\begin{tabular}{|c|c|c|c|c|c|}
\hline Terremoto & M & $I_{o}$ & Componente & $\underset{\left(\mathrm{cm} / \mathrm{sec}^{2}\right)}{A}$ & $\underset{\left(\mathrm{cm}^{2} / \mathrm{sec}^{3}\right)}{P}$ \\
\hline Mignano $21 / 11 / 70$ & 3,27 & V-VI & $\begin{array}{l}\text { SE-NW } \\
\text { NE-SW }\end{array}$ & $\begin{array}{l}16,8 \\
14,8\end{array}$ & $\begin{array}{r}108,7 \\
77,9\end{array}$ \\
\hline Mignano $26 / 11 / 70$ & 3,15 & $\mathrm{~V}$ & $\begin{array}{l}\text { SE-NW } \\
\text { NE-SW }\end{array}$ & $\begin{array}{r}9,3 \\
10,5\end{array}$ & $\begin{array}{l}24,3 \\
36,5\end{array}$ \\
\hline Mignano $\quad 6: 1 / 71$ & 3,20 & $\mathrm{~V}$ & $\begin{array}{l}\text { NE-SW } \\
\text { SE-NW }\end{array}$ & $\begin{array}{l}10,0 \\
11,5\end{array}$ & $\begin{array}{l}31,8 \\
22,7\end{array}$ \\
\hline Valfabbrica $14 / 2 / 71$ & 4,00 & VI.VII & $\begin{array}{l}\text { NE-SW } \\
\text { SE-NW }\end{array}$ & $\begin{array}{l}78,9 \\
49,2\end{array}$ & $\begin{array}{l}914,7 \\
361,4\end{array}$ \\
\hline
\end{tabular}


Si puó notare una maggiore uniformità tra i valori relativi alle due componenti delle registrazioni eflettuate a Mignano rispetto a quelia effettuata a Valfabbrica, dove è evidente una polarizzazione delle onde (Fig. 1).

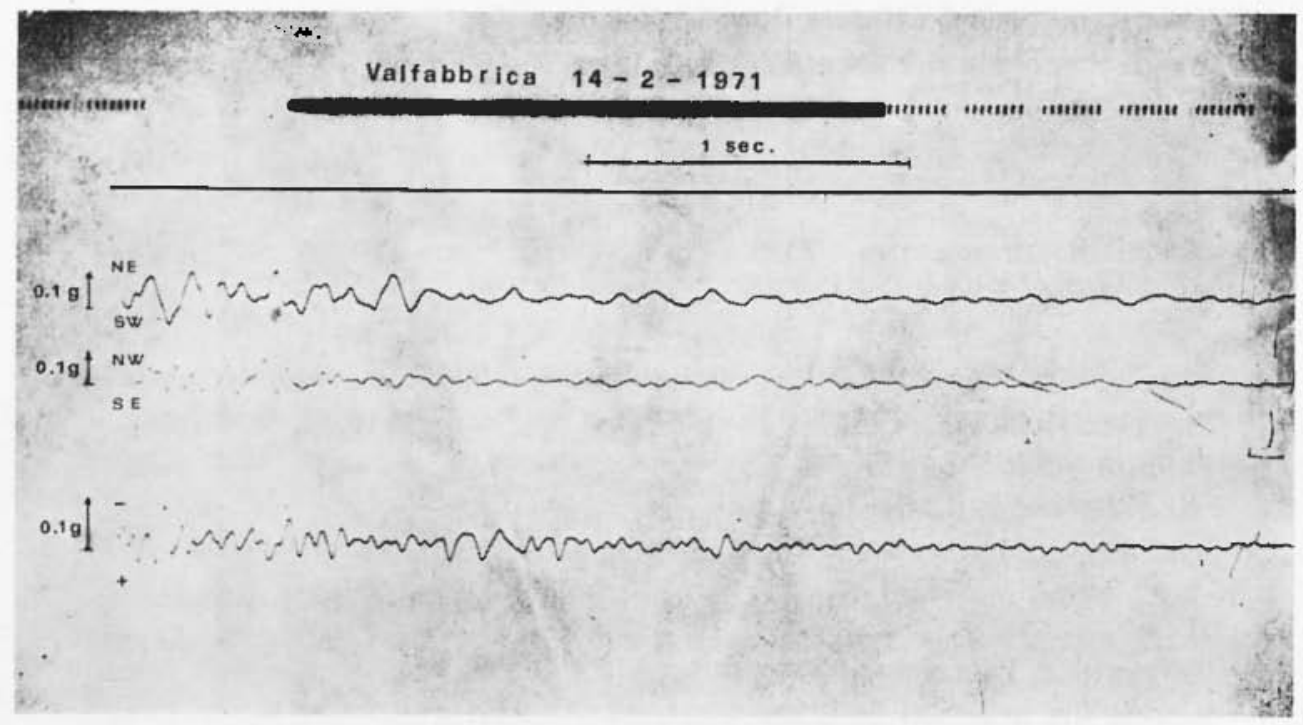

Fig. 1

3. - SPETTRI Ji RISPOSTA.

Seguendo la teoria classica degli spettri di risjosta, deseritta in una precelente memoria, abbiamo elaborato gli spettri per ciaseuna delle otto componenti a nostra disposizione. Allo scopo di risparmiare il tempo impiegato sul calcolatore abbiamo scelto un incremento del periodo $T$ non costante, diradando i punti nella zona meno significativa, ed abbiamo ripetuto il calcolo per tre valori dello smorzamento.

Le Figure 2-9 si riferiscono agli spettri disegnati da un "plotter" collegato all'elaboratore elettronico ehe ha eseguito i calcoli. Le leggere diflerenze che si possono riseontrare tra i primi quattro diagrammi e quelli riportati nell'articolo citato sono dovute ad un miglioramento nella determinazione della linea di zero.

Prima di ottenere i valori medi dello spettro di risposta occorre procedere alla normalizzazione delle curve, cioè riportarle a valori uguali 
per le intensità. A tale scopo lefiniamo l'intensità spettrale come l'area contenuta dalla curva dello spettro di velocità fra i periodi 0,1 sec e 2,5 sec:

$$
S I_{h}=\int_{0.1}^{2.5} S_{\nu}(h, T) d T .
$$

Applicando tale formula otteniamo i risultati della Tabella M.

Tabella 11

\begin{tabular}{|c|c|c|c|c|}
\hline Terremoto & Componente & $S I_{0}$ & $S I_{0.1}$ & $S I_{0.2}$ \\
\hline Mignano 21:11:70 & $\begin{array}{l}S E-N W \\
N E-S W\end{array}$ & $\begin{array}{l}4,233 \\
4,240\end{array}$ & $\begin{array}{l}2,753 \\
2,735\end{array}$ & $\begin{array}{l}2,301 \\
2,189\end{array}$ \\
\hline Mignano $26 / 11 / 70$ & $\begin{array}{l}S E-N W \\
N E-S W\end{array}$ & $\begin{array}{l}2,877 \\
2,874\end{array}$ & $\begin{array}{l}1,957 \\
2,086\end{array}$ & $\begin{array}{l}1,619 \\
1,804\end{array}$ \\
\hline Mignano $6 ! 1 / 71$ & $\begin{array}{l}\mathrm{NE}-\mathrm{SW} \\
\mathrm{SE}-\mathrm{NW}\end{array}$ & $\begin{array}{l}2,292 \\
2,445\end{array}$ & $\begin{array}{l}1,532 \\
1,668\end{array}$ & $\begin{array}{l}1,243 \\
1,480\end{array}$ \\
\hline Valfahbrica $14 / 2 / 71$ & $\begin{array}{l}\mathrm{NE}-\mathrm{SW} \\
\mathrm{SF}-\mathrm{SW}\end{array}$ & $\begin{array}{l}6,994 \\
3,237\end{array}$ & $\begin{array}{l}5,822 \\
2,538\end{array}$ & $\begin{array}{l}\overline{5}, 556 \\
2,4 \overline{5} 3\end{array}$ \\
\hline
\end{tabular}

Dividendo le ordinate delle curve relative a ogni componente per $l_{a}$ propria intensità spettrale, e moltiplicancolole per l'intensità della prima, ne otteniamo i valori normalizzati. Nell'eseguire i calcoli abbiamo distinto la media dei soli dati di Mignano (Fig. 10) da quella generale (Fig. 11).

Dallo spettro medio di valocità, merliante la formula

$$
S_{a}=\frac{2 \pi}{T} \tilde{\omega}_{v},
$$

otteniamo quello dell'accelerazione (Figg. 12 e 13). 


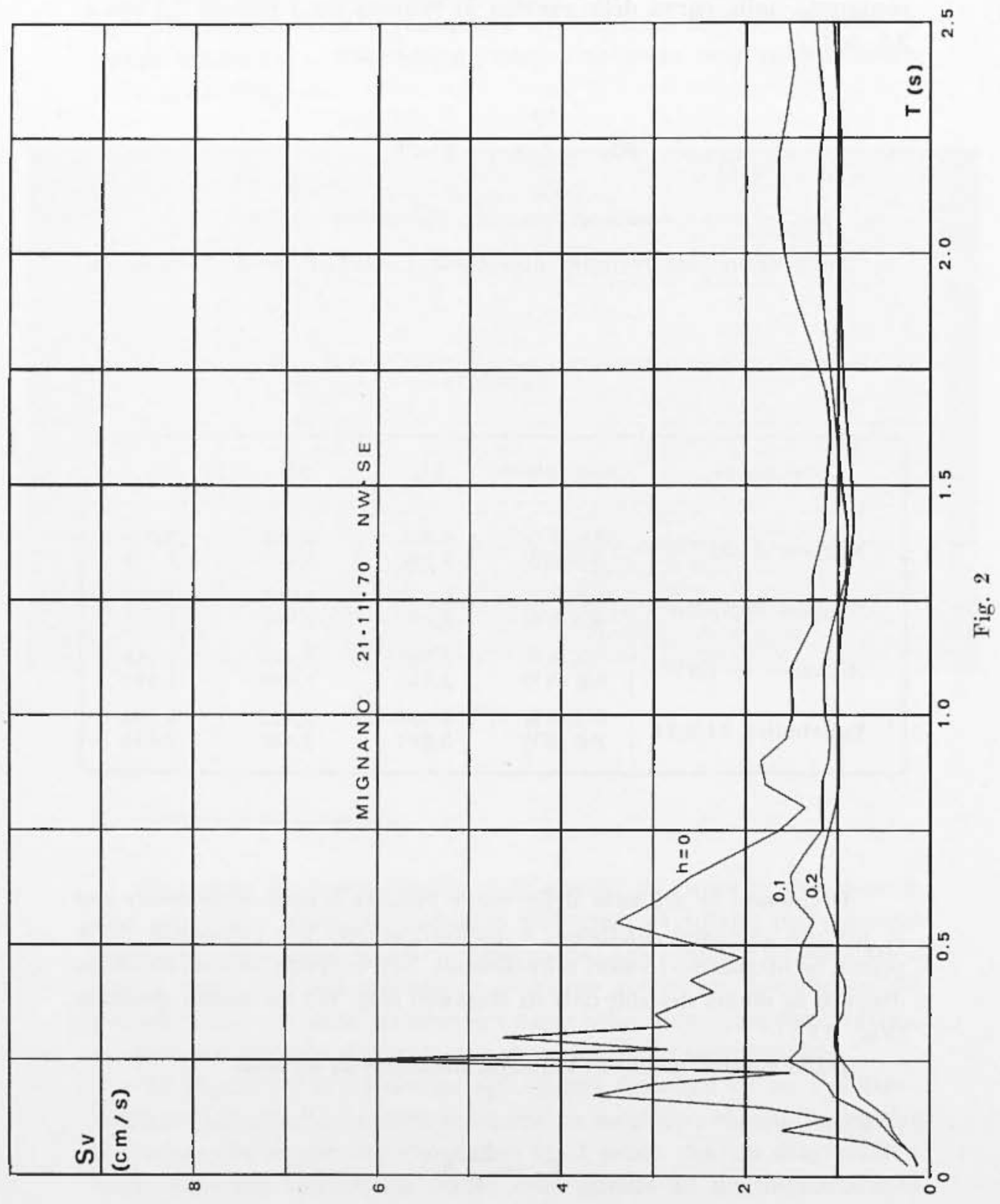




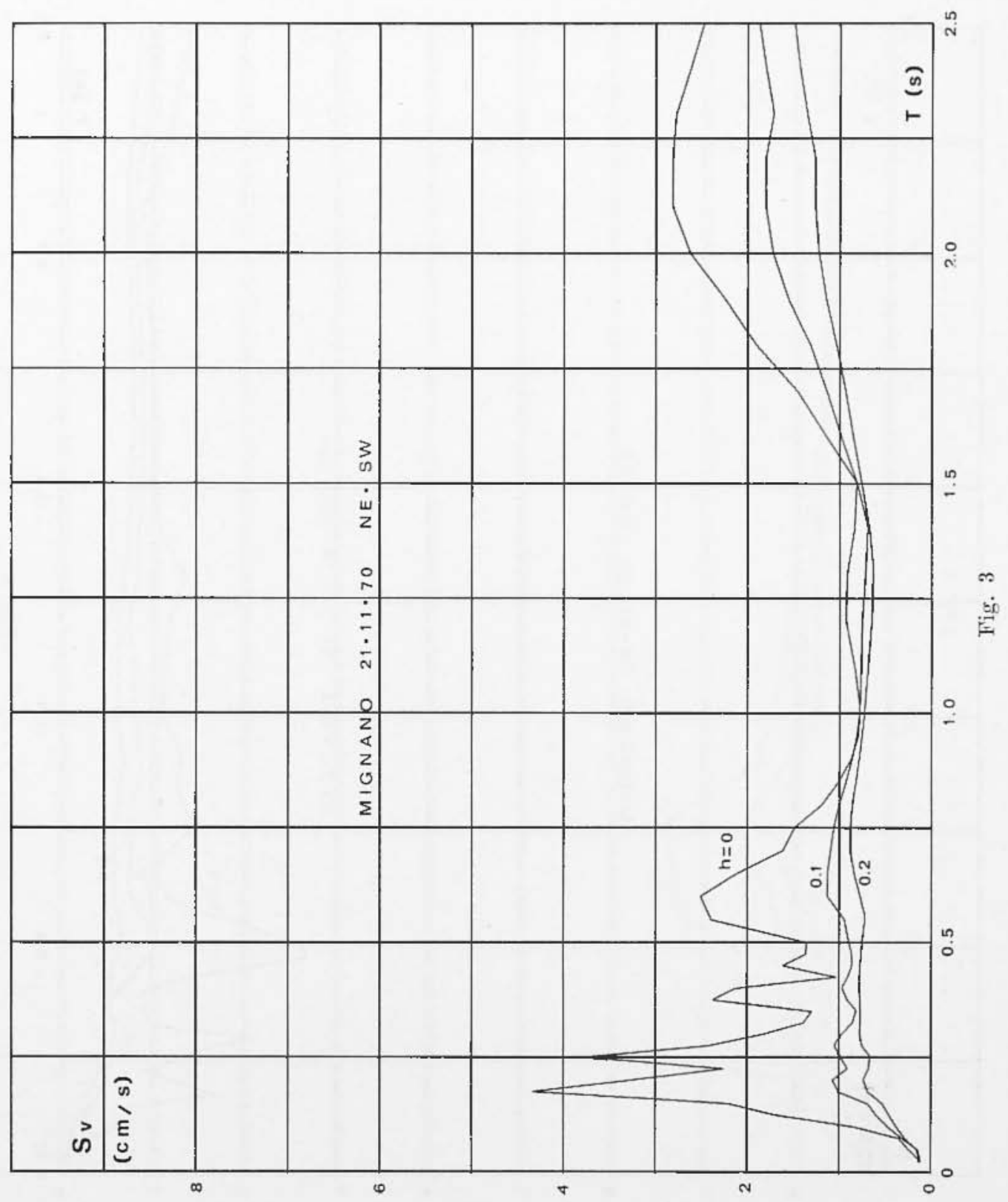




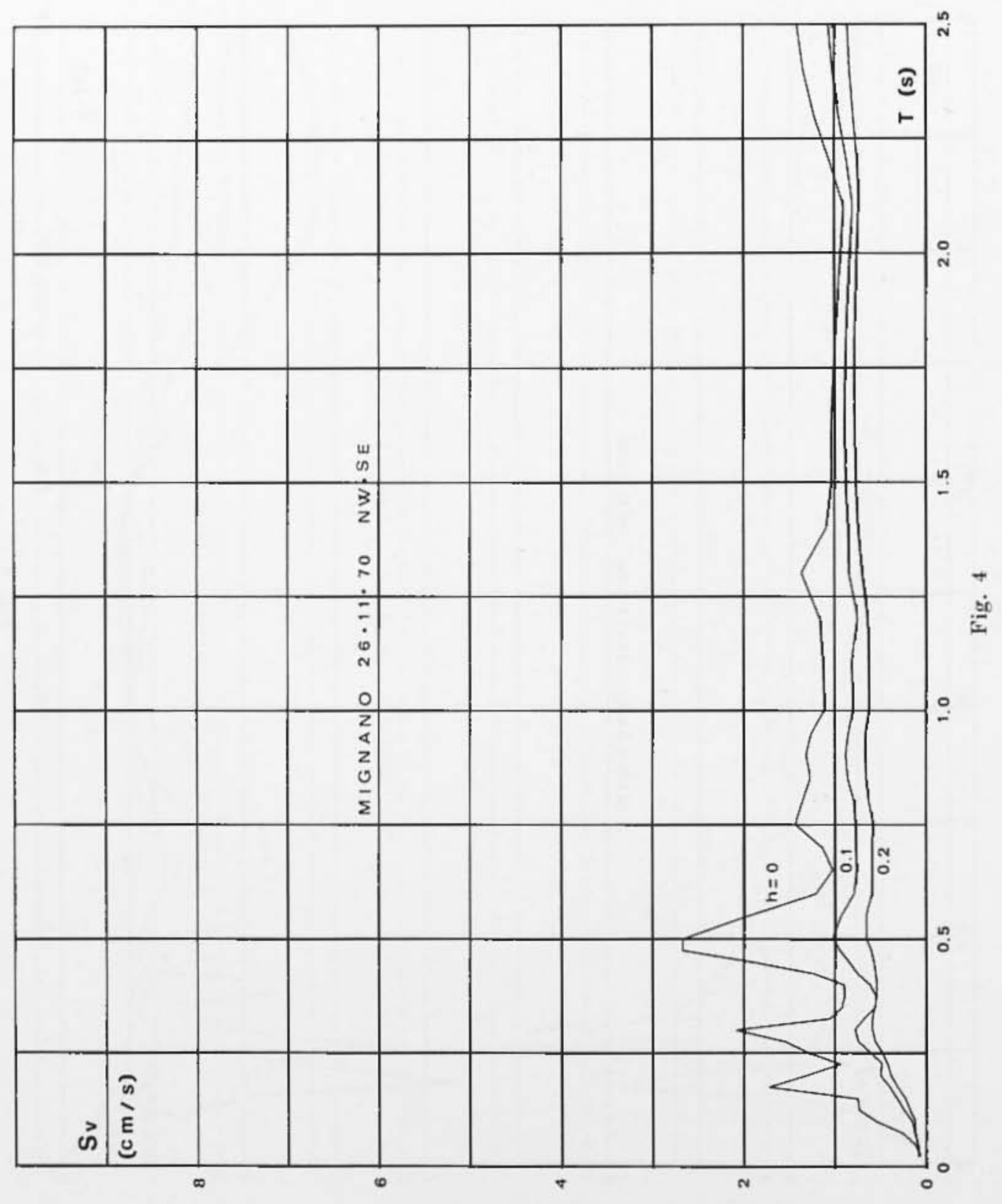




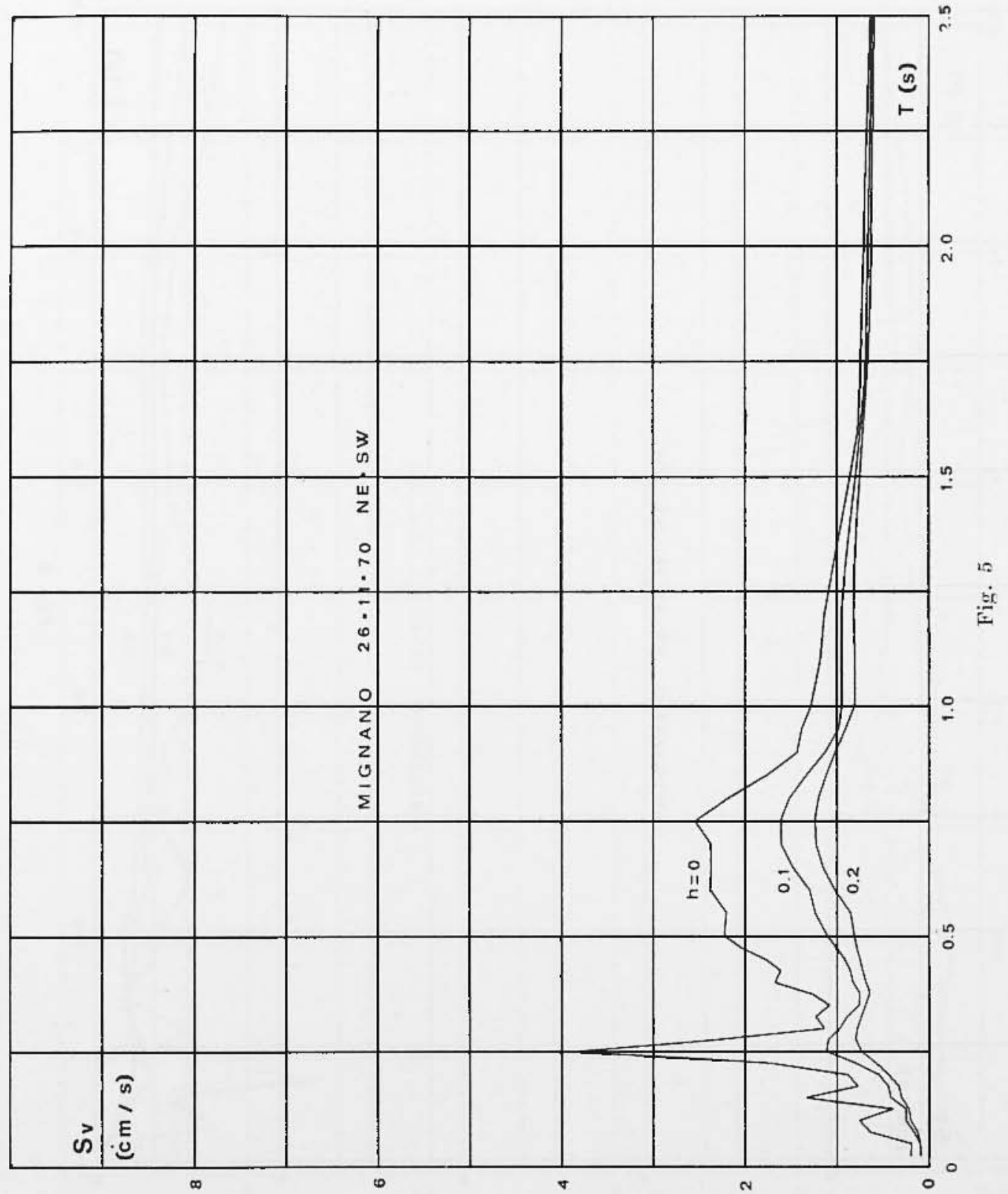




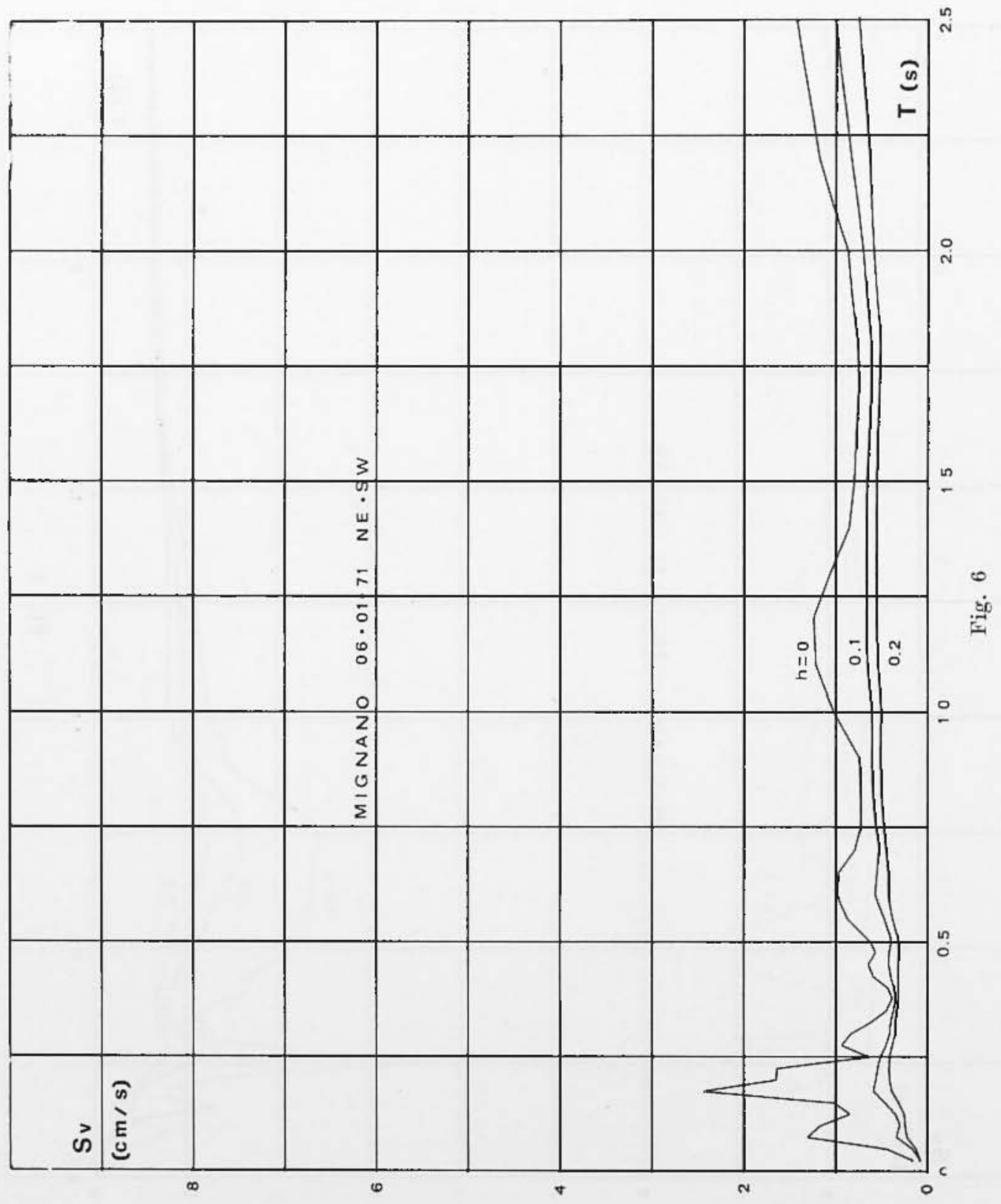




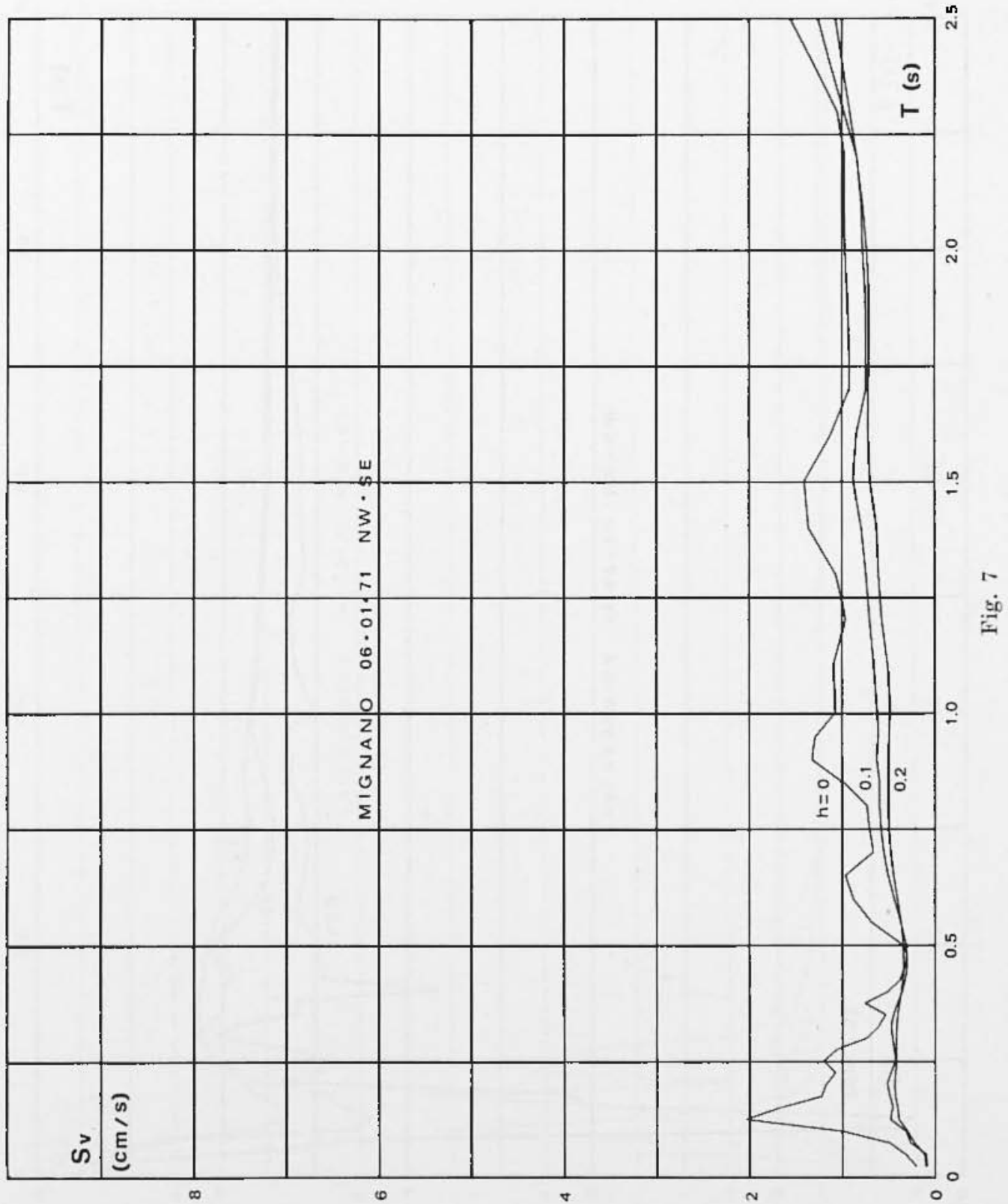




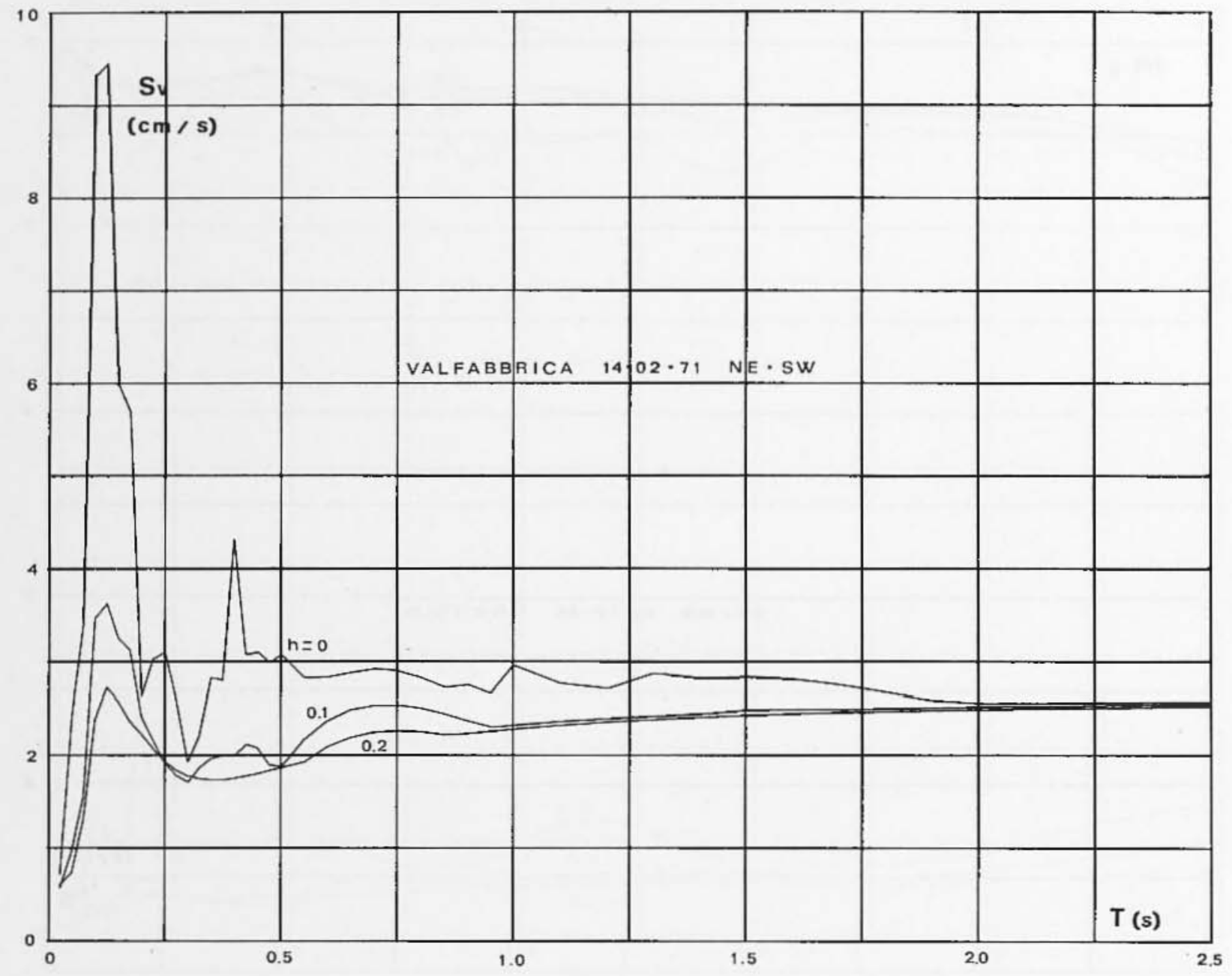

Fig. 8 


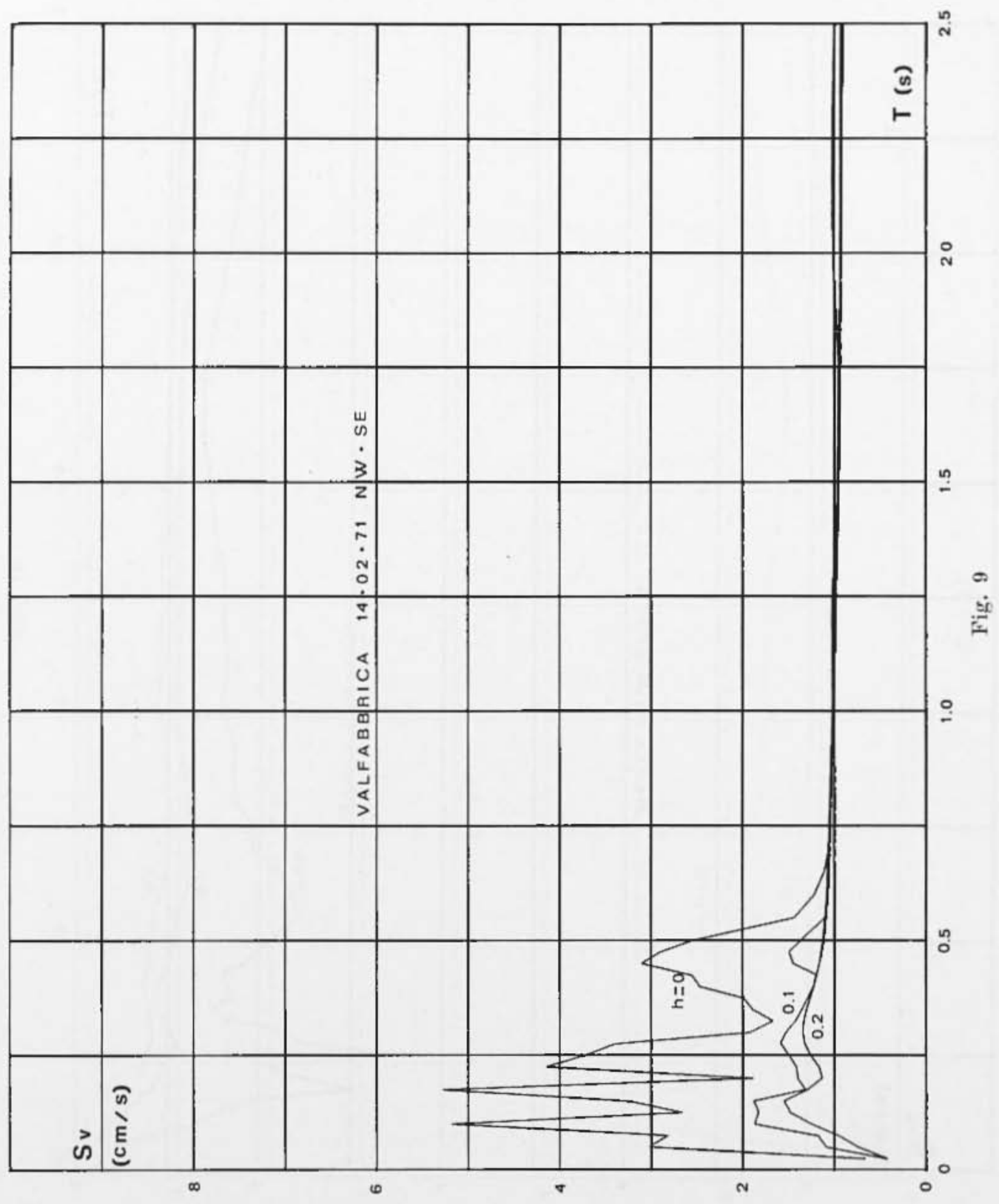




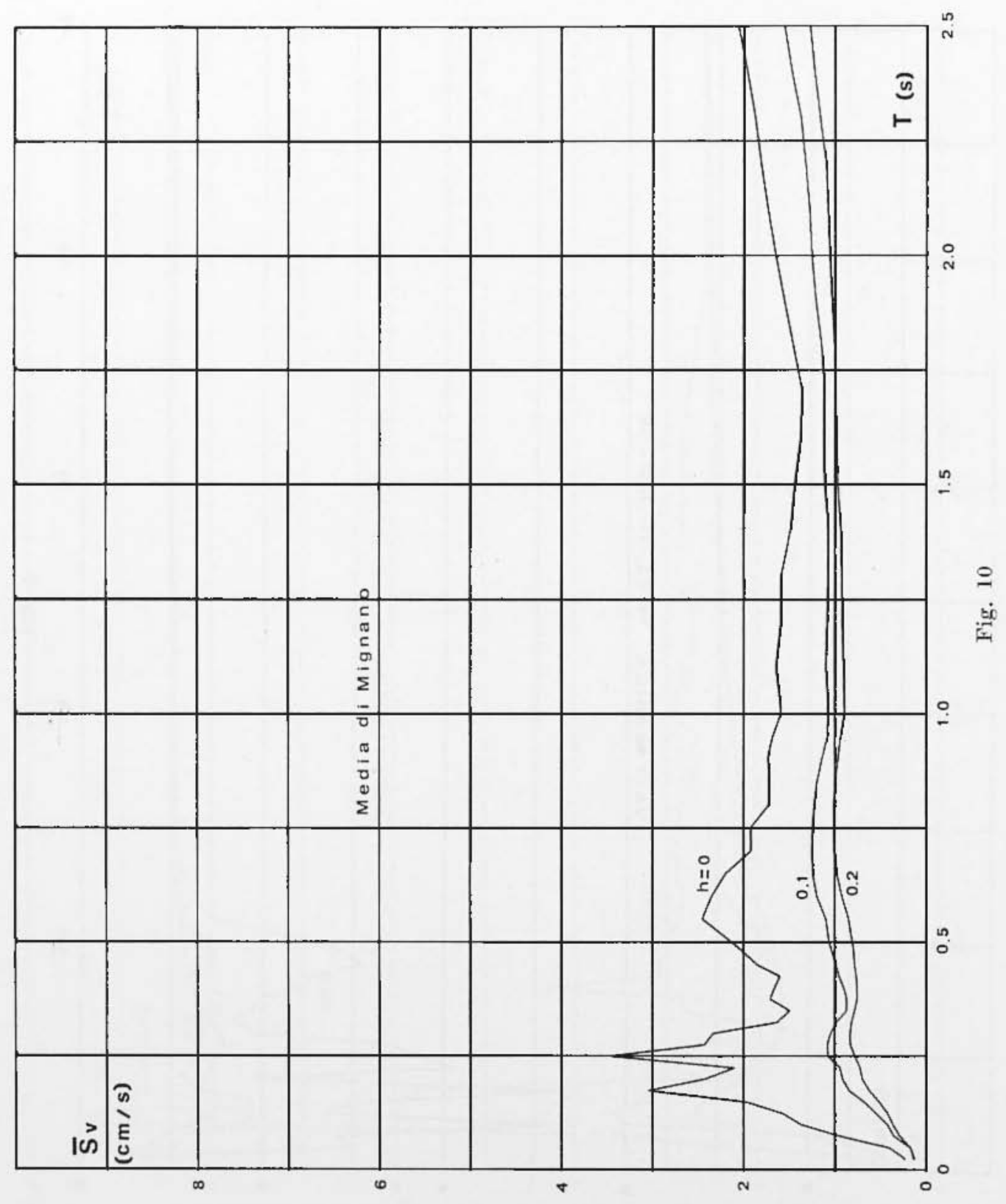


STUDJO DEGL ACCELEROGRAHW OTTWYTTI XEI RECENTI EVEXTI SISWICI

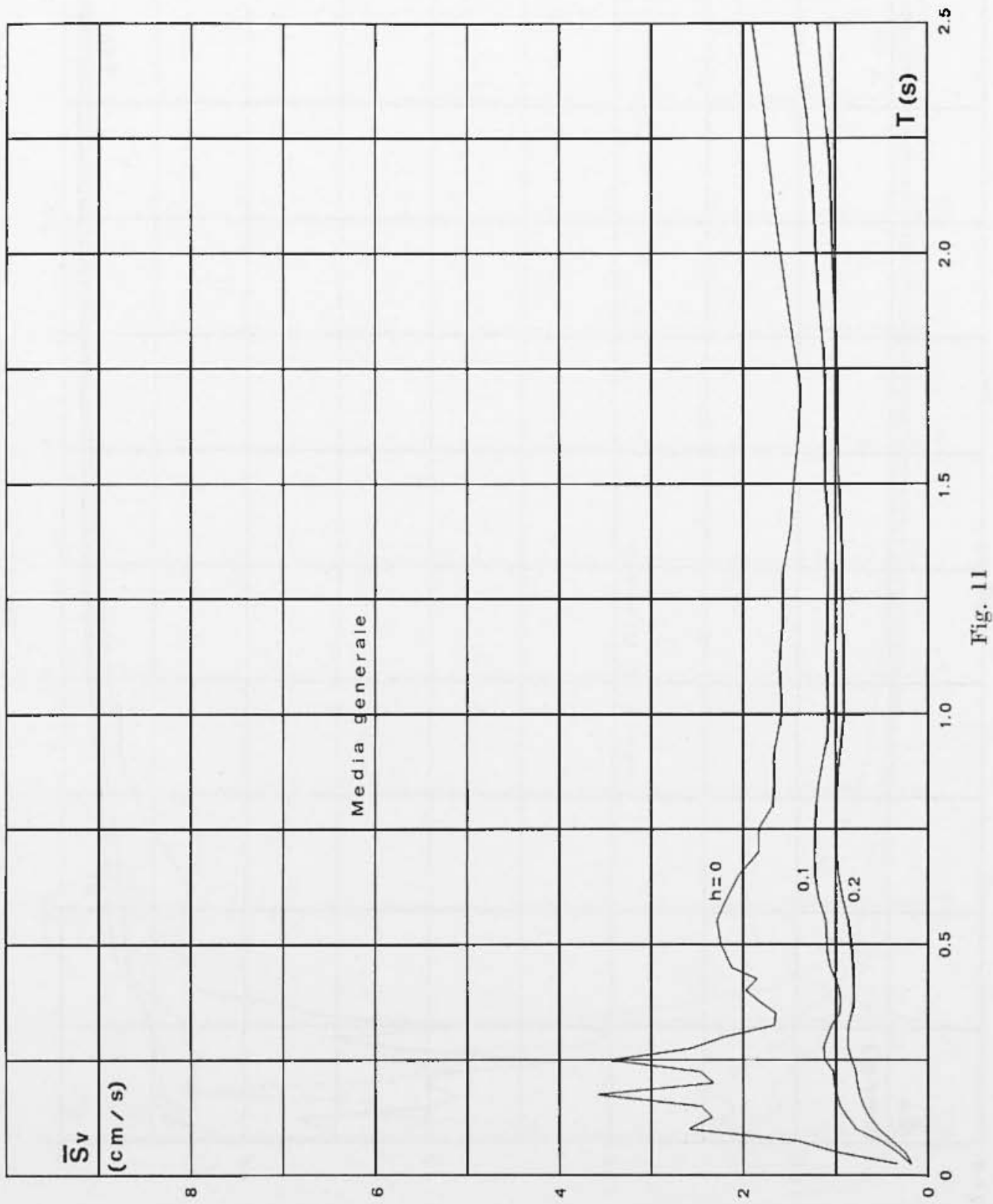




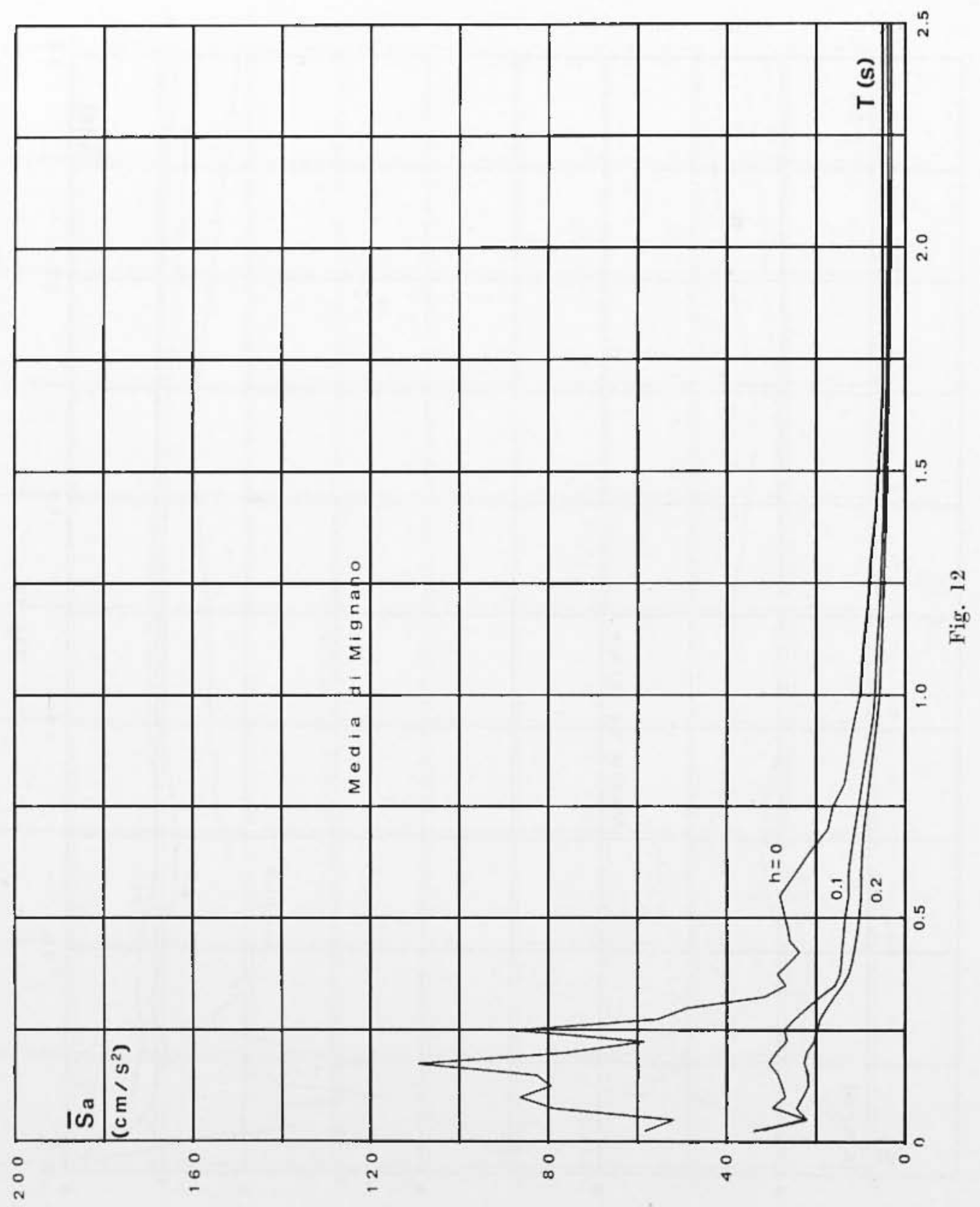




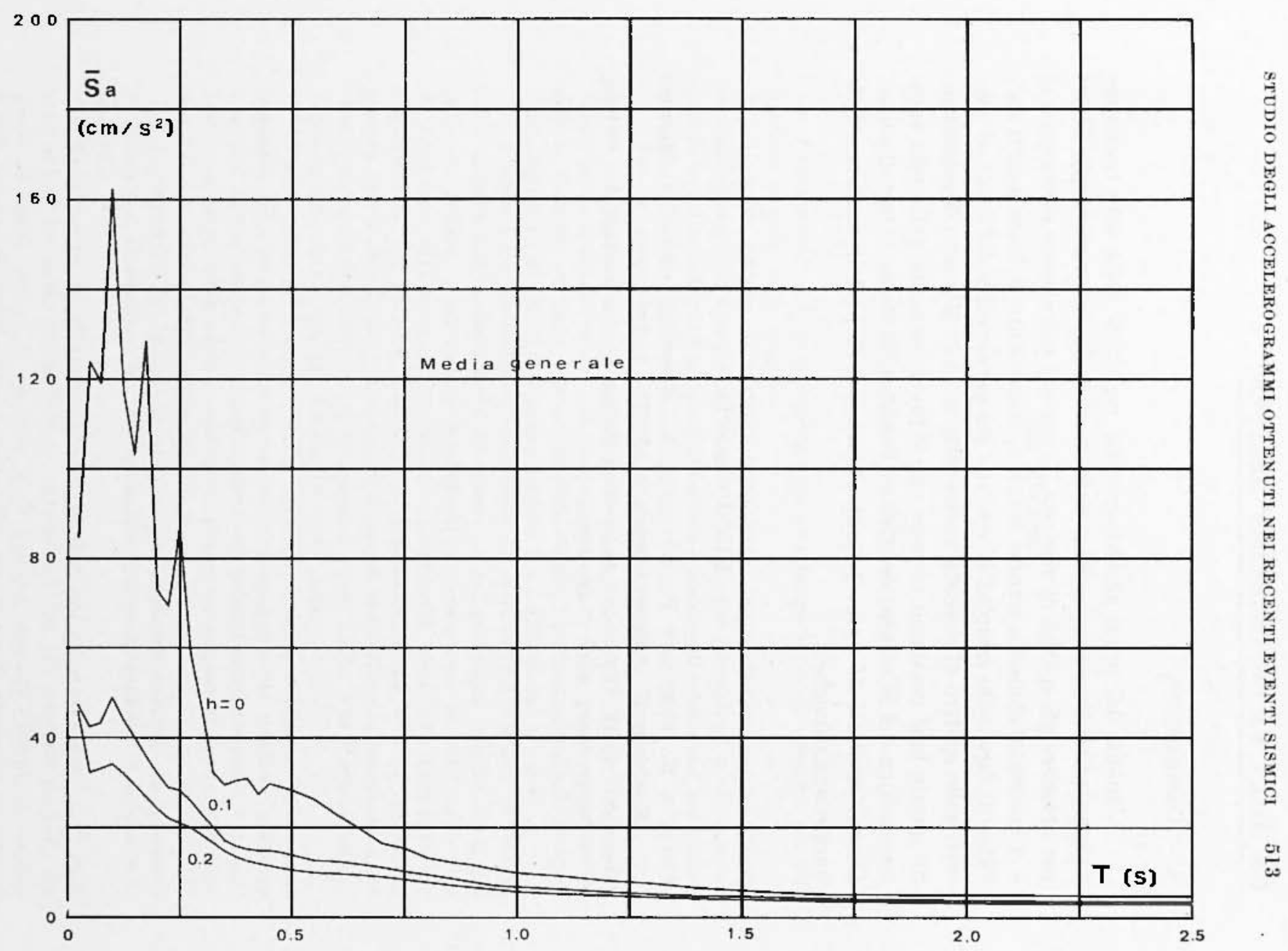

Fig. 13 


\section{1. - CoNGLUSIONI.}

Laualisi rei primi accelerogrammi registrati dalla rete nazionale Tha consentito di provare su diati resli i metodi di elaborazione piis adatti per ottenere gli spet tri di risposta. L'uso alel calcolatore elettronico si i. dimostrato idoneo a ruesto scopo e, nonostante si fosse partiti dall'idea di fare delle semplici prove, si è grià pervenuti a dei risultati concreti sullo spettro di risposta medio nella zona di Mignano Montelungo. Su queste basi possiamo ritencre cle il funzionamento della rete acelerometrica del Ministero dei Isavori Pubblici, in corso di installazione, fornirà risultati di notevole importanza per il perfezionamento delle norme antisismiche.

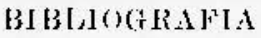

Coxsole R., Peteoxagt F., Soxagtia A., Fenomeni sismici a Mignano Montelungo, "Ann, di Geofisica", XXIV, 1, 1971.

Jousner G. W., Spetrum Analysis of Sirong-Motion Earhiquakes. "Bull, Steism. Soe. Anı.", 43, 1.953. 